Donald A. Donahue, Jr., DHEd, MBA, MSJ, FACHE, FRSPH

\section{BAHAMAS DISPATCHES}

"Change is the only constant in life" (Heraclitus).

$\mathrm{D}$ isaster medicine and public health demand the ability to change and adjust to the needs of the population. With this special section, DMPHP embarks on change to reinforce and enhance the relevance of the journal. There is great value in the critical analysis of disaster health science, a purpose to which this journal has been dedicated since its inception.

There is equal value, we believe, in the examination of the actions and activities in the immediate aftermath of a disaster. This not only informs retrospective analysis, but can contribute to immediate and short term planning for effective response.

Herein, we present several brief "reports from the field" on the response to Hurricane Dorian in the Bahamas. All cyclones are punishing, but Dorian was particularly savage. For nearly 24 hours, Dorian and its $200 \mathrm{mph}$ (322 km/hr) winds-the equivalent of an EF-5 tornado-hovered over the island of Abaco. As is the norm in disaster, the brunt of the impact was borne by the socio-economically disadvantaged.

Dorian was not so much an anomaly as further evidence of a changing world. Earlier this year, DMPHP focused on serial hurricanes, prompted by the devastation from hurricanes Irma and Maria. It is clear that humanity will continue to face weather events of increased intensity and unprecedented destruction. As a peer reviewed source, DMPHP will deliver more timely insights into significant events. In this manner, the distinct body of knowledge on disaster medicine and global health will be expanded, with the goal of enhanced global health security. As
Thomas Hobbes wrote in Leviathan in 1668, Scientia potentia est.

\section{CHANGES TO DMPHP}

Since its very first edition, Disaster Medicine and Public Health Preparedness has welcomed input from around the globe. Catastrophe knows no political boundary, and the lessons shared within these pages have universal applicability. At the same time, the frequency and severity of disasters continue to accelerate. No place on earth has been immune from unprecedented impacts of nature. In addition, the potential for human-inflicted damage-whether by acts of malevolence or by accident—shows no evidence of abating.

In light of this, the editorial leadership of DMPHP has initiated a series of subtle but meaningful actions. Over the coming months, the editorial board that has so positively supported this publication will evolve to be more globally representative.

As this edition of the journal goes to press, the details of establishment of an Asian editorial office, headquartered at Tianjin University in the Peoples Republic of China, are being finalized. Discussions are ongoing with multiple parties in Europe, the Indian Ocean region, the Middle East, South America, and elsewhere to expand and enhance global representation.

This reorganization is designed to better reflect the needs of specific areas, generally aligned with the World Health Organization's six regional groupings, and to enrich the flow of knowledge world-wide. The journal will continue to be an important voice for promoting global health security. Our goal is to strengthen both the science of disaster medicine and the inclusivity of its practice. 